\title{
THE EFFECTS OF TEST INTERPRETATION STYLES AND THE STATUS OF TESTS IN CAREER COUNSELLING
}

\author{
NELIA FRADE \\ Learning Centre \\ University of Johannesburg \\ GIDEON P DE BRUIN \\ deondb@uj.ac.za \\ Department of Human Resource Management \\ University of Johannesburg
}

\begin{abstract}
The effects of two styles of test interpretation, namely directive and collaborative, and clients' perceptions of the technical status of tests, namely high and low, were compared for 32 postgraduate psychology students who served as career counselling clients. Clients who received a collaborative interpretation perceived their counsellor as more attractive and trustworthy than did clients who received a directive test interpretation. Interpretation style did not have an effect on session impact. Clients' perceptions of test status had a noticeable, but statistically non-significant effect on counsellor evaluations and session impact. Implications for test-interpretation practice are discussed.
\end{abstract}

Key words: Career counselling, testing interpretation, collaborative counselling

Psychological testing of abilities, personality traits and types, and vocational interests has traditionally played an important role in the career counselling literature. Much research effort has focused and continues to focus on demonstrating the validity of measures of these attributes as predictors of occupational choice and vocational adjustment (Walsh \& Betz, 1995; Wampold, 2001). Overall, it appears that these measures have some predictive validity (Foxcroft, 2001).

While relatively more is known about how to construct psychological measures for career counselling purposes and how to validate these measures, much less is known about how to best interpret the results and how to best give feedback to clients (Zytowski, 1999). For much of the $20^{\text {th }}$ century, the socalled trait-and-factor approach to career counselling appeared to be the most popular approach (Sharf, 2002). The basis of this approach is that (a) individuals should know themselves in regard to their abilities, personality traits, vocational interests, and vocational values, (b) individuals should know the world of work concerning aspects such as the structure of employment, the availability of employment opportunities in a particular field, and the future prospects in a particular field, and (c) a match between a person's attributes and specific work environments should be made so that the needs of the individual and the needs of the work environment are best met (Holland, 1997).

The stereotypical view of this approach to career counselling is that the client consults a career counsellor who administers a battery of psychological tests and on the basis of the test results, makes a recommendation about fields of work or study most appropriate for the client. This approach to career counselling has been caricatured as "two interviews and a cloud of dust" (Brown \& Krane, 2000). Although it is not always made explicit, the counsellor is likely to adopt the role of an expert who decides on the tests to be included in the test battery. The counsellor then interprets the tests, gives feedback to clients, and makes recommendations. All this occurs without much input on the part of the client (Campbell, 2000). It should be noted that what occurs in practice may differ substantially from the stereotypical view described above. It is more likely that skilled career counsellors, who work from a trait-and-factor approach, will have involved their clients in some aspects of the career counselling and test interpretation process.

Career counselling is an activity that influences a wide range of people, from adolescents to adults. Contemporary writers, for example Brown and Brooks (2002) and Feller (2001), increasingly emphasise the view that career development is a life span issue affecting other life roles. Given this, these writers advocate that career counselling should equip the client with the necessary skills to make career decisions. They contend that individuals can only make sensible career decisions when career counsellors facilitate conditions that enable clients to gain greater selfknowledge and career knowledge (Sharf, 2002).

To this end, career counsellors frequently use psychological tests, an advocated practice, because tests assist a person in discovering information about him or herself, which is relevant to eventual career choice (Zunker, 2002). In addition, psychological tests help the person determine relative strengths and weaknesses, promote a more scientific orientation and provide ways to support and confirm what otherwise might have been speculation (Brown \& Krane, 2000). Furthermore, since career counsellors regularly use psychological tests to aid them in their therapeutic practice, it is imperative that these tests be of high technical status. This implies that they are: (a) reliable and valid, (b) that the item content and norms are appropriate, and (c) that the test instructions and interpretation methods are clear (Foxcroft, 2001).

The dearth of literature concerning the dissemination of test results in career counselling, compels counsellors to rely on their theoretical orientations when interpreting test results (Zytowski, 1999). Thus, using theoretical orientation as a point of departure, it can be said that trait-and-factor counsellors, such as Dorn (1988), appear to advocate a passive role for the client during the process of test interpretation. The counsellor, on the other hand, adopts an expert role and assumes responsibility for the interpretation of psychological tests (Brown \& Brooks, 2002). Super's (1983) approach, however, appears to differ in that the client is encouraged to assume an active role in the interpretation and dissemination of test results. From this perspective, a collaborative relationship emerges in which both the counsellor and the client assume responsibility for the counselling process (Super, 1983).

Finn and Tonsager (1992) share Super's approach to test interpretation. They contend that the client should not be seen as a passive target of the social influence power exhibited by the counsellor and the psychological test. Instead, the client should actively participate in the test interpretation process. However, this can only be achieved if the counsellor cultivates a climate of active participation in the interpretation process. Hence, active participation depends on the counsellor adopting a non-delivered or collaborative style of psychological test interpretation (Duckworth, 1990). 
Goodyear (1990) provides an explanation of a collaborative approach to career counselling, where the client is involved in every possible way. For example, the counsellor clarifies the goals of testing for the client, explaining what is being measured and the purpose of the measurement. Following this, the counsellor, together with the client, selects the appropriate tests (Campbell, 2000). Once the test information is available, the counsellor, adopting a tentative and questioning approach rather than a directive approach (Maree \& Ebers hn, 2002), assists the client in exploring the meanings of the results in relation to the purpose of counselling.

In an attempt to shed light on the question of client involvement in the process of test interpretation, Hanson, Claiborn and Kerr (1997), conducted a study in which clients were randomly assigned to one of two test interpretation styles. Accordingly, interpretations were either delivered (non-interactive) to the client, with minimal client involvement in the process, or conducted interactively (nondelivered), with considerable client involvement. Hanson et al. (1997) revealed that the interactive (non-delivered) counselling style was evaluated as having more impact and that counsellors were perceived as more influential on the combined dimensions of expertness, trustworthiness and attractiveness.

Despite these promising preliminary results, no consensus has been reached concerning the most effective method for test interpretation. The research problem of this study is therefore based on the need to investigate how assessment results should be communicated to facilitate appropriate interpretation in the career counselling process. In addition, since psychological tests play an integral role in the career counselling process, the study highlights the importance for psychological tests to be of high technical status (Foxcroft, 2001). The aim of this study, then, was to investigate the differential effects of two test interpretation styles, namely delivered (non-interactive) and collaborative (interactive), and the status of tests, namely high and low, in career counselling.

In this study, a social influence perspective was used to examine how participants respond to the test interpretations received in career counselling (cf. Heppner \& Dixon, 1985). Interpretations were either delivered to the participant, with minimal participant involvement in the process, or conducted in a collaborative style, with considerable participant involvement. Participants evaluated the session and the counsellor after an introductory session and a feedback session. It was hypothesised that participants would consider counsellors using a collaborative test interpretation style to be more influential on the dimensions of expertness, trustworthiness and attractiveness than would counsellors using a delivered test interpretation style. It was also hypothesised that participants would view collaborative test interpretation as having more impact than delivered interpretation, where impact is measured in terms of session Depth, Smoothness, Positivity and Arousal (Hanson et al., 1997). Similarly, it was hypothesised that counsellors, adopting a high status test, would be more influential on the dimensions of expertness, trustworthiness and attractiveness and thus, have more impact, where impact is measured by Depth, Smoothness, Positivity and Arousal, than would counsellors adopting a low status test. Lastly, it was hypothesised that there is an interaction effect between test interpretation style and test status (Foxcroft, 2001).

\section{RESEARCH DESIGN}

\section{Research approach}

The study employed a quantitative randomized pre-test post-test experimental design.

\section{Research Method}

\section{Participants}

Participants were 32 postgraduate students in psychology at a university in Johannesburg (six men and twenty six women; thirty white and two black). The counsellors, one man and one woman, were professional counselling psychologists with four and eight years of experience, respectively, in the field of career counselling. Participants were volunteers who were recruited from postgraduate psychology classes at a university in Johannesburg.

\section{Instruments}

Self-Directed Search (SDS) was used to assist participants to locate those occupations that best matched their interests and abilities. The SDS utilizes a broad range of self-ratings to assess the participant's resemblance to each of Holland's six personality types, namely Realistic (practical), Investigative (scientific), Artistic, Social, Enterprising (business) and Conventional (clerical). These six personality types can be linked to six working environments. Upon completion of this instrument, an individual is assigned a three-letter code. Internal consistency coefficients for the SDS range from 0.90 to 0.93 , whilst test-retest reliabilities range from 0.76 to 0.89 (Zunker \& Osborn, 2002). In addition, concurrent validity ranges from $46.7 \%$ to $76.0 \%$, and predictive validity ranges from $39.6 \%$ to $79.3 \%$.

Counsellor Rating Form-Short Version (CRF-S) was used to measure participants' ratings of the counsellor with regard to three subscales: expertness, trustworthiness and attractiveness (Tracey, Glidden, \& Kokotovic, 1988). Each subscale consists of four items, which is scored by summing the item scores. Previously reported reliabilities (Cronbach's alpha) for the expertness subscale range from 0.84 to 0.93 , for the trustworthiness subscale from 0.79 to 0.92 , and for the attractiveness subscale from 0.84 to 0.92 (Corrigan \& Schmidt, 1983; Ellingson \& Galassi, 1995; Tracey et al., 1988; Ponterotto \& Furlong, 1985). The CRF-S uses a 7-point Likert scale anchored by the words "not very" (1) and "very" (7).

Session Evaluation Questionnaire Form 5 (SEQ) was used to measure the impact of the counselling session. The SEQ (Form 5) measures two independent evaluative dimensions of participants' perceptions of their sessions, namely Depth and Smoothness, and two dimensions of their post-session mood, namely Positivity and Arousal. Depth refers to a session's perceived power and value, whereas Smoothness refers to a session's comfort, relaxation and pleasantness. Positivity refers to feelings of confidence and clarity as well as happiness and absence of fear or anger, whereas Arousal refers to feeling active and excited as opposed to quiet and calm. Factor analytic studies have confirmed all four dimensions as underlying session ratings by counsellors and clients (Stiles \& Snow, 1984). The SEQ (Form 5) includes 21 items in a 7-point bipolar adjective format. Reliability, measured by coefficient alpha, has been high for all SEQ (Form 5) indices across a wide variety of conditions and settings, for example 0.90 for Depth and 0.93 for Smoothness (Reynolds, Stiles, Barkham, Shapiro, Hardy \& Rees, 1996).

\section{Experimental procedure}

Participants were randomly assigned to one of four experimental conditions, namely (a) directive counselling style and high status test $(n=8)$, (b) directive counselling style and low status test ( $n$ $=8$ ), (c) collaborative counselling style and high status test ( $n=$ 8 ), and (d) collaborative counselling style and low status test ( $n$ $=8$ ). All participants were requested to complete a consent form and demographic questionnaire. Upon completion of these, participants were escorted to either a directive counsellor or a collaborative counsellor.

Counsellors were trained in the use of the test to be interpreted, as well as in the directive and collaborative protocols. Furthermore, counsellors participated in role-plays to familiarise themselves with the counselling style they were expected to adopt in the experiment. Each counsellor adopted only one counselling style throughout the study. The sessions were videotaped to ensure that only those sessions that adhered to the counselling style required were included in the study. 
Participants took part in two half-hour counselling sessions. The first session was aimed at establishing rapport with the participant and determining their motivation for participating in the study. After the first session, participants rated the counsellor using the CRF-S and the session using the SEQ (Form 5). Participants then completed the SDS, but prior to receiving feedback about their scores on the SDS, the perceived status of the inventory was manipulated. In the second session, each participant received feedback from either the directive or collaborative counsellor. After a participant had received feedback he or she was requested to again complete the CRF-S and SEQ (Form 5). It should be noted that the participant received feedback from the counsellor he or she encountered in the first session.

\section{Independent variables (experimental manipulations)}

Test feedback style. Counsellor protocols were developed by the researcher and were identical except for the style of feedback (directive or collaborative) and the perceived status of the test (high or low). The protocol for the directive style of test interpretation consisted of: (a) the counsellor stating that test results would be interpreted for the participant by the counsellor, (b) the counsellor discussing the three highest scores on the SDS and relating them to relevant occupations, and (c) the counsellor terminating the session by summarising the test results and offering no new information.

The protocol for the collaborative style of test interpretation consisted of the following elements: (a) the counsellor stating that the participant would be involved in the interpretation of test results, (b) the counsellor asking the participant to note scores that were significantly high or low as depicted by the SDS, (c) the counsellor explaining the results of the SDS and enquiring whether the participant agrees with the results, (d) the counsellor asking the client to give examples of how the results agree with his or her daily activities, (e) the counsellor affirming or modifying the test results in accordance with the client's reaction, (f) the counsellor terminating the session by asking the participant to summarise his or her own test results. For example, the counsellor could ask, "What are some conclusions about yourself that you could draw from these test results?"

Test status. Test status was manipulated by the researcher who either informed a participant that the SDS had a high technical status and was highly successful in predicting occupational outcomes (high status) or that the SDS, although widely used, was not a perfect instrument and that it was not a perfect predictor of occupational outcomes (low status). Participants who were informed that the SDS had a low technical status were debriefed before the study ended.

\section{Experimental manipulation check}

Eight postgraduate psychology students who did not participate in the counselling sessions, were requested to view recorded sessions on videotape and to rate them according to four 7-point Likert-type items. The purpose of these ratings were to ascertain whether the manipulation of counselling style in the experiment was successful and whether the two counselling styles were perceived as sufficiently different.

Raters firstly indicated whether the participant in the counselling session was actively (1) or passively (7) involved in the counselling process, secondly whether the counsellor assumed an expert (1) or collaborative (7) role, thirdly whether the counsellor's comments were phrased tentatively (1) or decisively (7), and fourthly whether the counsellor's comments were in the form of questions (1) or statements (7).

These students were purposefully selected as raters because of their training in counselling skills which allowed them to ascertain if differences in counselling style existed or not. Results of the manipulation check showed that the raters perceived the two counselling styles as clearly different. Participants in the collaborative condition were rated as being more actively involved, whereas those in the directive condition were rated as being more passively involved $[t(14)=12.77, p<0.01]$. The counsellor in the collaborative condition was rated as more collaborative, whereas the counsellor in the directive condition was rated as assuming a more expert role $[t(14)=-12.48, p<$ $0.01]$. In addition, the counsellor in the collaborative condition was rated as phrasing comments more tentatively, whereas the counsellor in the directive condition was rated as phrasing comments more decisively [ $t(14)=-10.42, p<0.01]$. In the last place, the counsellor in the collaborative condition was rated as phrasing more comments in the form of questions, whereas the counsellor in the directive condition was rated as phrasing more comments in the form of statements [ $t(14)=-10.36, p<0.01]$. On the basis of these results it can be said that the counselling style variable was manipulated as intended and that it reflected what it was intended to reflect.

\section{RESULTS}

The means and standard deviations of the CRF-S and the SEQ (Form 5) are summarised in Table 1, which reveals a pattern where participants in the collaborative condition appeared to give more favourable ratings on the CRF-S and the SEQ (Form 5). Similarly, participants in the high perceived test status condition appeared to give more favourable ratings with regard to the CRF$S$ and the SEQ (Form 5).

TABLE 1

DESCRIPTIVE STATISTICS FOR THE CRF-S AND THE SEQ (FORM 5)

\begin{tabular}{|c|c|c|c|c|c|c|c|c|}
\hline & \multicolumn{4}{|c|}{ Directive Style } & \multicolumn{4}{|c|}{ Collaborative Style } \\
\hline & \multicolumn{2}{|c|}{ Pre-test } & \multicolumn{2}{|c|}{ Post-test } & \multicolumn{2}{|c|}{ Pre-test } & \multicolumn{2}{|c|}{ Post-test } \\
\hline & $\mathbf{M}$ & SD & $\mathbf{M}$ & SD & $\mathbf{M}$ & SD & $\mathbf{M}$ & SD \\
\hline \multicolumn{9}{|l|}{ Low Status } \\
\hline \multicolumn{9}{|l|}{ CRF-S } \\
\hline Expertness & 21.25 & 2.66 & 22.62 & 2.32 & 24.75 & 2.25 & 25.62 & 1.84 \\
\hline Trustworthiness & 22.00 & 2.00 & 23.25 & 2.12 & 26.12 & 1.88 & 26.62 & 1.60 \\
\hline Attractiveness & 22.62 & 2.82 & 22.00 & 3.02 & 25.25 & 1.58 & 25.87 & 1.35 \\
\hline \multicolumn{9}{|l|}{ SEQ (Form 5) } \\
\hline Depth & 3.75 & 0.59 & 4.72 & 1.02 & 4.87 & 0.69 & 5.85 & 0.52 \\
\hline Smoothness & 5.37 & 1.13 & 5.55 & 0.48 & 6.30 & 0.68 & 6.50 & 0.55 \\
\hline Positivity & 4.55 & 1.01 & 5.15 & 0.79 & 5.92 & 0.63 & 6.22 & 0.82 \\
\hline Arousal & 3.27 & 0.41 & 3.75 & 1.03 & 3.50 & 0.52 & 4.05 & 0.88 \\
\hline \multicolumn{9}{|l|}{ High Status } \\
\hline \multicolumn{9}{|l|}{ CRF-S } \\
\hline Expertness & 21.88 & 4.01 & 22.25 & 3.37 & 21.62 & 4.10 & 23.12 & 5.30 \\
\hline Trustworthiness & 21.62 & 3.62 & 22.87 & 2.64 & 23.50 & 2.00 & 24.37 & 2.56 \\
\hline Attractiveness & 22.00 & 3.42 & 22.50 & 2.26 & 24.25 & 1.39 & 24.87 & 1.72 \\
\hline \multicolumn{9}{|l|}{ SEQ (Form 5) } \\
\hline Depth & 4.32 & 0.53 & 4.95 & 0.76 & 4.75 & 1.31 & 5.05 & 1.36 \\
\hline Smoothness & 5.55 & 1.29 & 5.95 & 0.81 & 5.72 & 1.11 & 5.87 & 1.31 \\
\hline Positivity & 5.17 & 0.81 & 5.47 & 0.78 & 5.70 & 0.84 & 5.92 & 0.85 \\
\hline Arousal & 3.90 & 0.86 & 3.87 & 0.84 & 3.70 & 0.78 & 4.20 & 0.86 \\
\hline
\end{tabular}

Note. All values rounded to two decimal places.

\section{Counsellor Rating Form-Short Version (CRF-S)}

The post-test CRF-S subscale scores were subjected to a $2 \times$ 2 MANCOVA, with pre-test subscale scores on the CRF-S as covariants. The independent variables were test feedback style (directive and collaborative) and perceived test status (low status and high status). A significant main effect was observed for test feedback style, $[F(3,23)=3.16$, Wilks' $\left.\Lambda=0.78, \eta^{2}=0.23, p=0.44\right]$, but not for perceived test status, $\left[F(3,23)=0.62\right.$, Wilks' $\left.\Lambda=0.93, \eta^{2}=0.08, p=0.61\right]$. 
In addition, the interaction of test feedback style and test status was non-significant, $[F(3,23)=0.163$, Wilks' $\Lambda=$ $\left.\left.0.98, \eta^{2}=0.02, p=0.92\right)\right]$. Test feedback style accounted for approximately $23 \%$ of the variance in the linear combination of the post-test CRF-S scales, test status for approximately $8 \%$ and the interaction of test feedback style and test status for approximately $2 \%$.

Following the significant main effect for test feedback style, separate ANCOVA's were conducted with each one of the CRF$S$ subscales in turn serving as dependent variable and test feedback style as independent variable. Statistically significant differences between the two groups were observed with regard to attractiveness, $\left[F(1,25)=7.42, \eta^{2}=0.23, p=0,01\right]$, and trustworthiness, $\left[F(1,25)=4.46, \eta^{2}=0.15, p=0,04\right]$, but not for expertness, $\left[F(1,25)=3.86, \eta^{2}=0.13, p=0.06\right]$. Inspection of the means in Table 1 shows that participants in the collaborative condition rated the counsellors higher on the dimensions of attractiveness and trustworthiness. After controlling for pre-test differences, test feedback style accounted for approximately $23 \%$ of the variance in attractiveness, $15 \%$ of the variance in trustworthiness and $13 \%$ of the variance in expertness.

\section{Session Evaluation Questionnaire (Form 5)}

Post-test SEQ (Form 5) subscale scores were also subjected to a $2 \times 2$ MANCOVA, with pre-test subscale scores on the SEQ (Form 5) as covariants. No statistically significant main effects were observed for test feedback style, $[F(4,21)=0.21$, Wilks' $\left.\Lambda=0.962, \eta^{2}=0.04, p=0.93\right]$, for perceived test status, $\left[F(4,21)=0.58\right.$, Wilks' $\left.\Lambda=0.90, \eta^{2}=0.10, p=0.68\right]$, or their interaction, $\left[F(4,21)=0.59\right.$, Wilks' $\Lambda=0.90, \eta^{2}=0.10, p=$ $0.67]$. Style accounted for approximately $4 \%$ of the variance in the linear combination of the post-test SEQ (Form 5) scales, where status accounted for $10 \%$ and their interaction for a further $10 \%$. Given the lack of statistically significant relations in the MANCOVA, no follow-up univariate analyses were done.

\section{DISCUSSION}

The findings of the present study concur with previous research showing that counsellors with a collaborative test interpretation style are perceived as more influential on the combined dimensions of expertness, attractiveness and trustworthiness on the CRF-S (cf. Hanson et al., 1997). From a social influence perspective on career counselling (cf. Heppner \& Dixon, 1985), these findings possibly indicate that clients like, admire and trust counsellors with a collaborative style more than they like, admire and trust counsellors with a directive style. This may hold important implications for counselling outcomes as it suggests that clients are more likely to form strong working alliances with counsellors that they like, admire and trust.

We anticipated that the collaborative test interpretation style would lead to more favourable ratings with reference to session Depth and Smoothness and greater feelings of Positivity and Arousal, but found that test interpretation style had little effect on client's session evaluations. This result is similar to that of Hanson et al. (1997), who reported non-significant effects concerning session Smoothness, Positivity and Arousal. Hanson et al. (1997), however, did find that a collaborative style was associated with greater session Depth. Taken together, these results might suggest that test interpretation style does not have a major impact on session evaluations.

Although statistically non-significant, test status did have noticeable effects on counsellor ratings (approximately $8 \%$ of the variance accounted for) and session evaluations (approximately $10 \%$ of the variance accounted for), which have implications for practice. Moreover, the interaction of test status and test interpretation style accounted for a further $10 \%$ of the variance in session evaluations. These results lead us to theoretically speculate that client perceptions of the status and quality of the tests used in career counselling may have an effect on their evaluations of counselling sessions. It appears that clients may respond more positively to career counselling sessions if they perceive the tests and instruments used to be of high status and quality.

The results of this study have implications for practice. Firstly, if a collaborative style leads to more favourable attitudes towards the counsellor, then the working alliance between client and counsellor may possibly be strengthened by adopting a collaborative style. Seen against the background of the importance of the working alliance as a critical ingredient in successful counselling (Wampold, 2001) it appears prudent to recommend that counsellors adopt a collaborative rather than a directive test interpretation style.

Secondly, if a client perceives that a test utilized within the process of career counselling has a high status and is useful, such a client may possibly be more likely to act on the outcomes of the session and to commit to further active participation in counselling sessions. This may be seen as a desired outcome and it appears prudent to recommend that counsellors communicate or demonstrate in counselling sessions their confidence and trust in the tests used. From a collaborative counselling perspective, this confidence and trust should focus on the quality of the information that the tests provide. Interpreting and giving meaning to the information remains the joint task of the client and counsellor.

It is appropriate to highlight three limitations of the study. Firstly, although a sample size of thirty-two participants cannot be regarded as small in experimental psychotherapy or counselling studies, it did mean that the study lacked the statistical power to render noticeable effects ( $10 \%$ of the variance of the dependent variable accounted for remained non-significant). However, given the increasing number of methodologists who recommend that statistical significance testing should be regarded as of lesser importance (e.g. Kline, 2004), or even banned from psychological research (e.g. Hunter, 1997; Schmidt, 1996), it is noteworthy that relatively large effect sizes that are consistent with previous research, have been obtained in this study.

Secondly, external validity may have been compromised because all participants were postgraduate psychology students of which $94 \%$ were white and $81 \%$ were women. It is not clear that other samples would respond in similar ways to the interventions and more research concerning this is necessary. In addition, since each counsellor adopted only one counselling style throughout the study, the results may reflect an interaction between gender of the counsellor and the counselling style. Further research in the area should focus on eliminating gender as a confounding variable.

Thirdly, the manipulation of test status was done by a credible third party (the first author) and not by the counsellors themselves. It is possible that the effect of perceived test status may have stronger counselling implications if clients base their perceptions on the views and behaviours of the counsellors rather than a third party. This should be examined in further research.

In conclusion, the results suggest that a client's perceptions of the quality of tests used in the career counselling process may influence his or her evaluations of career counselling sessions. A potentially important consequence of this is that it may be beneficial for the counselling process if counsellors emphasise the status and quality of the tests that they use. However, most importantly, the results of this study suggest that a counsellor's test interpretation style can make a difference to how favourably the counsellor is perceived by the client in the career counselling situation. From a social influence perspective, a collaborative 
counselling style, where the counsellor actively involves the client in the interpretation process, appears more appropriate as favourable counsellor perceptions may enhance the working alliance between client and counsellor.

\section{REFERENCES}

Brown, D., Brooks, L., \& Associates (2002). Career choice and development ( $4^{\text {th }}$ ed.). San Francisco, CA: Jossey-Bass.

Brown, S. D., \& Krane, N. E. R. (2000). Four (or five) sessions and a cloud of dust: Old assumptions and new observations about career counselling. In S. D. Brown \& R. W. Lent (Eds), Handbook of counseling psychology (pp. 740-766). New York: Wiley.

Campbell, V. L. (2000). A framework for using tests in counselling. In C. E. Watkins \& V. L. Campbell (Eds), Testing and assessment in counseling practice (pp. 3-11). Mahwah, NJ: Erlbaum.

Corrigan, J. D., \& Schmidt, L. D. (1983). Development and validation of revisions in the counsellor rating form. Journal of Counseling Psychology, 30, 64-75.

Dorn, F. J. (1988). Utilizing social influence in career counseling: A case study. Career Development Quarterly, 36, 269-280.

Duckworth, J. (1990). The counselling approach to the use of testing. The Counseling Psychologist, 18, 198-204.

Ellingson, K. T., \& Galassi, J. P. (1995). Testing two theoretical explanations for the attraction-enhancing effects of selfdisclosure. Journal of Counseling and Development, 73, 535541.

Feller, R. W. (2001). Theoretical voices directing the career development journey: Holland, Harris-Bowlsbey, and Krumboltz- JoAnn Harris-Bowlsbey, John Krumboltz, John Holland. Retrieved June 30, 2005 from the World Wide Web: http://www.findarticles.com/p/articles/mi_m0JAX/is_3_49/ ai_72703619.

Finn, S. E., \& Tonsager, M. E. (1992). Therapeutic effects of providing MMPI-2 test feedback to college students awaiting therapy. Psychological Assessment, 4, 278-287.

Foxcroft, C (2001). Developing a psychological measure. In C. Foxcroft, \& G. Roodt (Eds.), An introduction to psychological assessment in the South African context (pp. 69-85). Oxford University Press.
Goodyear, R. K. (1990). Research on the effects of test interpretation: A review. The Counseling Psychologist, 18, 240-257.

Hanson, W. E., Claiborn, C. D., \& Kerr, B. (1997). Differential effects of two test-interpretation styles in counselling: A field study. Journal of Counseling Psychology, 44, 400-405.

Heppner, P. P., \& Dixon, D. N. (1985). A review of the interpersonal influence process in counselling. The Personnel and Guidance Journal, 59, 542-550.

Holland, J. L. (1997). Making vocational choices: A theory of personalities and work environments ( $3^{\text {rd }}$ ed.). Odessa, FL: Psychological Assessment Resources.

Maree, J. G., \& Ebers hn, L. (2002). Lifeskills and career counselling. Sandown: Heinemann.

Ponterotto, J. G., \& Furlong, M. J. (1985). Evaluating counselor effectiveness: A critical review of rating scale instruments. Journal of Counseling Psychology, 32, 597-616.

Reynolds, S., Stiles, W. B., Barkham, M., Shapiro, D. A., Hardy, G. E., \& Rees, A. (1996). Acceleration of changes in session impact during contrasting time-limited psychotherapies. Journal of Consulting and Clinical Psychology, $64,577-586$.

Sharf, R. S. (2002). Applying career development theory to counseling. ( $3^{\text {rd }}$ ed.). Pacific Grove: Brooks/Cole.

Stiles, W. B., \& Snow, J. S. (1984). Dimensions of psychotherapy session impact across sessions and across clients. British Journal of Clinical Psychology, 23, 59-63.

Super, D. E. (1983). Assessment in career guidance: Toward truly developmental counseling. The Personnel and Guidance Journal, 61, 555-562.

Tracey, T. J., Glidden, C. E., \& Kokotovic, A. M. (1988). Factor structure of the Counselor Rating Form-Short. Journal of Counseling Psychology, 35, 330-335.

Wampold, B. E. (2001). The great psychotherapy debate: models, methods and findings. Mahwah, NJ: Erlbaum.

Zunker, V. G., \& Osborn, D. S. (2002). Using assessment results for career development. ( $6^{\text {th }}$ ed.). USA: Brooks/Cole.

Zunker, V. G. (2002). Career counseling: Applied concepts of life planning. ( $6^{\text {th }}$ ed.). USA: Brooks/Cole.

Zytowski, D. G. (1999). How to talk to people about their interest inventory results. In M. Savickas \& A. R. Spokane, (Eds.). Vocational interests: Their meaning, measurement, and counselling use. Palo Alto, CA: Davies-Blach. 